Result(s)* 311 patients with advanced EOC were included. 84 patients underwent PDS (27\%) and 227 IDS (73\%), respectively.

No residual disease was achieved in 258 out of 311 patients $(83 \%)$. Standard surgery was performed in 80 patients $(25.7 \%)$, radical surgery in 45 patients $(14.5 \%)$ and ultra-radical surgery in 186 patients $(59.8 \%)$. No residual disease was reached in 72 out of 80 standard surgery (90\%), 37 out of 45 radical surgery $(82.2 \%)$ and 149 out of 186 (80.1\%) ultraradical surgery.

Early and late severe (G3-4) complications were present in $32 / 311$ patients $(10.3 \%)$ and $23 / 311$ patients (7.4\%), respectively. Increased postoperative morbidity was directly related to the following perioperative factors: HIPEC [OR 4.4 (1.0$20)$ ], addition of each single surgical procedures to the standard surgery [OR $1.3(1.1-1.5)]$, number of surgical procedures added to modified pelvic posterior exenteration [OR 1.4 (1.12.0)], surgical complexity [SR vs $\mathrm{S}$ or R; OR 2.4 (1.1-5.7)].

In multivariate analysis, OS is not correlated with surgical complexity [OR 1.0(0.5-2.1)] and with the burden of early $[$ OR $0.7(0.3-1.5)]$ or late $[\mathrm{OR} 0.7(0.3-1.7)]$ severe complications.

Conclusion* Despite surgical timing, ultra-radical-surgery is needed to reach no residual disease in $>80 \%$ of our patients. The risk of early and late severe morbidities is increased for complex surgeries but they don't affect OS. Risk stratification should be used to plan perioperative care and best treatment.

\section{ERAS LEADS TO FEWER HOSPITAL COSTS IN ADVANCED OVARIAN CANCER SURGERY: A SECONDARY OUTCOME OF THE PROFAST TRIAL}

\begin{abstract}
${ }^{1} V$ Bebia Conesa*, 'S Rodríguez Conde, ${ }^{1} \mathrm{~PB}$ Asuncion, ${ }^{2} \mathrm{Y}$ Cossío Gil, ${ }^{1} \mathrm{~A}$ Gil-Moreno, ${ }^{2} J \mathrm{~L}$ Sánchez Iglesias. 'Hospital Universitari Vall d'Hebron, Gynecologic Oncology Unit, Barcelona, Spain; ${ }^{2}$ Hospital Universitari Vall d'Hebron, Information Systems, Barcelona, Spain
\end{abstract}

10.1136/ijgc-2021-ESG0.383

Introduction/Background* Enhanced recovery after surgery (ERAS) programs in advanced ovarian cancer (AOC) surgery have been developed in the last few years. The PROFAST randomized controlled trial showed that ERAS was associated with a decrease on length of stay and readmission. Here, we present the results of a secondary outcome, cost analysis, of the PROFAST trial.

Methodology The PROFAST trial was a prospective, interventional randomized clinical trial that enrolled women undergoing surgery for either suspected or diagnosed advanced ovarian cancer, at a reference hospital in gynecologic oncology in Barcelona (Spain). Patients were treated following either an ERAS protocol or conventional management (CM) protocol. The primary outcome was to evaluate reduction in length of stay (LOS). One of the secondary outcomes was to perform cost analysis betweem both groups.

Cost estimations were performed by two strategies: gross counting (cost of inpatient care, intensive care unit and surgery care), and microcosting (costs associated to image testing and laboratory). In both cases, costs were based on 2014 cost data. A sensitivity analysis of cost savings in different scenarios was performed. This trial was registered at ClinicalTrials.gov (NCT02172638).

Result(s)* From June 2014 to March 2018, 110 women were recruited, of which eleven were excluded. The ERAS group comprised 50 patients, and the CM group, 49 patients. LOS. Although mean overall cost per patient was higher in ERAS vs CM (14347 $€$ vs $12597 €)$, ERAS cost distribution was skewed by two patients that accounted for $48 \%$ of total intensive care unit costs. Comparing median costs, ERAS costs were significantly lower than CM costs $(7642 € /$ patient vs $8594 € /$ patient, $\mathrm{P}=.0275)$. Decrease in hospital readmission rates led to higher readmission costs in the $\mathrm{CM}$ group (Total costs $6126 € \mathrm{~V} 125725 €, \mathrm{P}=.028)$. Sensitivity analysis

\begin{tabular}{|cccc} 
Abstract 315 Table 2 & \\
\cline { 2 - 4 } & ERAS group & Conventional group & Difference \\
\hline Worst scenario & 9 median days & 12 median days & 3 median days \\
& $3,507 €$ & $4,654.01 €$ & $-\mathbf{1 1} 146.63 €$ \\
Best scenario & 5 median days & 7 median days & 2 median days \\
& $1,949 €$ & $2,714.84 €$ & $-\mathbf{7 6 6 . 3 0 €}$ \\
\hline
\end{tabular}

\begin{tabular}{|c|c|c|c|c|c|c|c|c|c|}
\hline \multirow[b]{2}{*}{ Resources } & \multicolumn{4}{|c|}{ ERAS (n: 50 patients) } & \multicolumn{5}{|c|}{ Conventional group (n: 49 patients) } \\
\hline & Total & Mean & Median & IQR & Total & Mean & Median & IQR & p-value* \\
\hline \multicolumn{10}{|l|}{ Cost in wards } \\
\hline First hospitalization in ward & $180.888 €$ & $3.618 €$ & $2.848 €$ & $2.085 €$ & $204.255 €$ & $4.169 €$ & $3.280 €$ & $2.193 €$ & 0,0588 \\
\hline Readmission day in ward & $5.894 €$ & $118 €$ & $0 €$ & $0 €$ & $59.303 €$ & $1.210 €$ & $0 €$ & $0 €$ & 0,0287 \\
\hline Total cost & $186.783 €$ & $3.736 €$ & $2.849 €$ & $2.098 €$ & $263.558 €$ & $5.379 €$ & $3.430 €$ & $3.021 €$ & 0,0143 \\
\hline \multicolumn{10}{|l|}{ Surgical costs } \\
\hline Primary surgical program & $195.669 €$ & $3.913 €$ & $3.638 €$ & $1.012 €$ & $184.997 €$ & $3.775 €$ & $3.697 €$ & $1.022 €$ & 0,3253 \\
\hline Reinterventions & $0 €$ & $0 €$ & $0 €$ & $0 €$ & $10.750 €$ & $219 €$ & $0 €$ & $0 €$ & 0,0771 \\
\hline Total cost & $195.669 €$ & $3.913 €$ & $3.638 €$ & $1.012 €$ & $195.747 €$ & $3.995 €$ & $3.697 €$ & $1.070 €$ & 0,2312 \\
\hline \multicolumn{10}{|l|}{ Image test and lab } \\
\hline Image test and lab at first hospitalization & $31.789 €$ & $662 €$ & $231 €$ & $499 €$ & $19.650 €$ & $401 €$ & $238 €$ & $468 €$ & 0,5421 \\
\hline Image test and lab at readmissions & $322 €$ & $6 €$ & $0 €$ & $0 €$ & $4.607 €$ & $94 €$ & $0 €$ & $0 €$ & 0,0455 \\
\hline Total cost & $32.111 €$ & $642 €$ & $205 €$ & $474 €$ & $24.257 €$ & $495 €$ & $265 €$ & $490 €$ & 0,1673 \\
\hline \multicolumn{10}{|l|}{ ICU cost } \\
\hline Total ICU cost at first hospitalization & $302.761 €$ & $6.582 €$ & $925 €$ & $350 €$ & $82.633 €$ & $1.836 €$ & $964 €$ & $233 €$ & 0,7209 \\
\hline Total ICU cost at readmissions & $0 €$ & $0 €$ & $0 €$ & $0 €$ & $51.065 €$ & $1.042 €$ & $0 €$ & $0 €$ & 0,151 \\
\hline Total cost & $302.761 €$ & $6.055 €$ & $900 €$ & $337 €$ & $133.698 €$ & $2.729 €$ & $964 €$ & $296 €$ & 0,6093 \\
\hline \multicolumn{10}{|c|}{ Total cost of inpatient resources } \\
\hline Total cost first hospitalizaton & $711.108 €$ & $14.222 €$ & $7.642 €$ & $3.062 €$ & $491.535 €$ & $10.031 €$ & $8.236 €$ & $4.015 €$ & 0,089 \\
\hline Total cost readmissions & $6.216 €$ & $124 €$ & $0 €$ & $0 €$ & $125.725 €$ & $2.569 €$ & $0 €$ & $0 €$ & 0,0287 \\
\hline Total cost & $717.324 €$ & $14.347 €$ & $7.642 €$ & $3.062 €$ & $617.260 €$ & $12.597 €$ & $8.594 €$ & $4.693 €$ & 0,0275 \\
\hline
\end{tabular}


showed that, in the worst predicted scenario (median LOS corresponding to 12 days), $1146 € /$ patient would be saved if ERAS protocols were applied.

Conclusion* Besides of achieving lower LOS and fewer readmission rates, implementation of an ERAS program in AOC surgery leads to valuable hospital savings. Therefore, ERAS should be the standard practice for AOC surgeries.

\section{ROLE OF INTEGRINS IN THE METASTATIC SPREAD OF HIGH-GRADE SEROUS OVARIAN CANCER}

${ }^{1} S$ Krajnak*, ${ }^{2} \mathrm{~J}$ Jäkel, ${ }^{1} \mathrm{~K}$ Anic, ${ }^{1}$ AS Heimes, ${ }^{1} \mathrm{~V}$ Linz, ${ }^{1} \mathrm{R}$ Schwab, ${ }^{1} \mathrm{E}$ Vacca, ${ }^{1} \mathrm{M}$ Schmidt, ${ }^{1} \mathrm{~A}$ Hasenburg, ${ }^{2} \mathrm{~W}$ Roth, ${ }^{1} \mathrm{~W}$ Brenner, ${ }^{1} \mathrm{M}$ Battista. 'University Medical Centre of the Johannes Gutenberg University Mainz, Department of Gynaecology and Obstetrics, Mainz, Germany; ${ }^{2}$ University Medical Centre of the Johannes Gutenberg University Mainz, Department of Pathology, Mainz, Germany

\subsection{6/ijgc-2021-ESG0.384}

Introduction/Background* In high-grade serous ovarian cancer (HGSOC) an early intraperitoneal metastatic spread is common which determines the therapeutical approach and prognosis. Integrins may be involved in metastatic spread of HGSOC. In this study, integrin expression was examined in primary tumour and metastases of HGSOC.

Methodology The expression of integrin $\alpha 2, \alpha 4, \alpha 5, \alpha 6$, and $\beta 1$ was assessed by immunostaining in tumour samples of the ovary, omentum, and peritoneum of each patient. Differences in integrin expression among tumour localisations and their association with clinicopathological parameters were examined by Fisher's exact test. The impact of integrin expression on progression-free survival (PFS) and overall survival (OS) was examined by Cox regression and Kaplan-Meier analyses.

Result(s)* 113 tumour samples of 40 HGSOC patients were examined. The expression of the integrins did not differ between the three tumour localisations (all p-values >0.05) with the exception of the expression of integrin $\beta 1$ in primary tumour and omentum $(77.5 \%$ versus $57.5 \%, p=0.014)$. Significant differences were also observed with respect to high expression of integrin $\alpha 4$ in primary tumour and omentum $(52.5 \%$ versus $47.5 \%, \mathrm{p}=0.008)$ and primary tumour and peritoneum $(52.5 \%$ versus $47.5 \%, \mathrm{p}=0.050)$. High expression of integrin $\alpha 4$ in peritoneum was associated with poorer PFS $(\mathrm{HR}=2.02 \quad 95 \%$ CI 1.01-4.05, $\mathrm{p}=0.047)$, younger age $(p=0.047)$ and death $(p=0.046)$. Median PFS in patients with

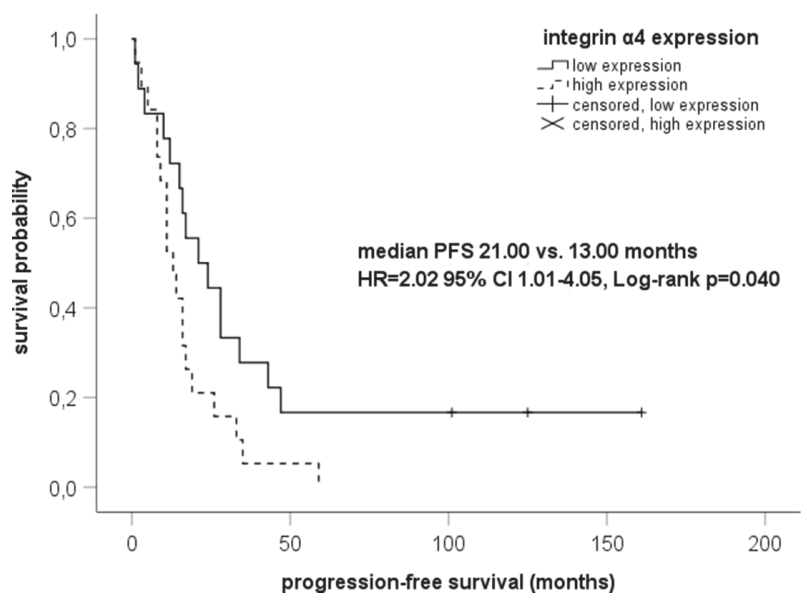

Abstract 317 Figure 1

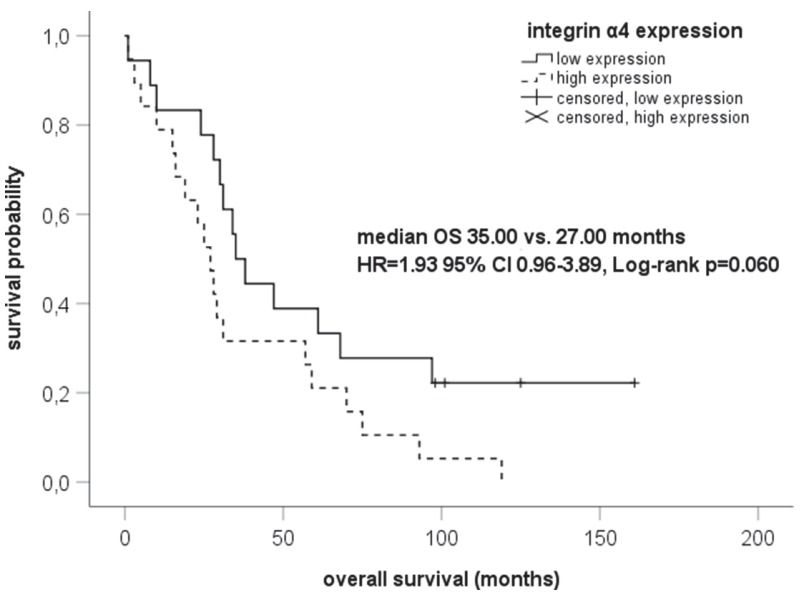

Abstract 317 Figure 2

high expression of integrin $\alpha 4$ was 13.00 months whereas median PFS in patients without high expression of integrin $\alpha 4$ was 21.00 months $(p=0.040)$. Expression of integrin $\alpha 2, \alpha 5$, $\alpha 6$, and $\beta 1$ did not correlate with PFS or OS.

Conclusion* Expression of integrin $\alpha 4$ may be altered during the metastasic spread of HGSOC and affect the prognosis. Expression of integrin $\alpha 2, \alpha 5, \alpha 6$, and $\beta 1$ did not reveal any prognostic value in HGSOC, even if expression of integrin $\beta 1$ differed between primary tumour and omental metastases.

\section{ADVANCED OVARIAN CANCER SURVIVAL RATES IN A MEDITERRANEAN POPULATION: AN 8-YEAR REAL- WORLD NATIONAL ANALYSIS OF THE MALTESE ISLANDS}

V Cassar*, MC Tabone, MP Agius, Y Muscat Baron. Mater Dei Hospital, Obstetrics and Gynaecology Department, MSD2090, Malta

\subsection{6/ijgc-2021-ESGO.385}

Introduction/Background* Surgical management of advanced ovarian cancer(AOC) is considered technically challenging. In order to achieve the required outcomes a centre requires expertise and flow of cases. ESGO recommends that accredited centres perform at least 24 complete cytoreductive(CCR) surgeries per year, with a minimum of 12 complete primary debulking surgeries(PDS).

Malta is a small European island with a population of 520,000 , seeing an average of 40 ovarian cancer cases per year, 20 of which are AOC. Being a small island, Malta faces a different reality and numerous challenges compared to other European countries.

Methodology This is a retrospective study, where all abnormal histology results suggestive of abnormal ovarian pathology during the time period of 2008-2016, processed by the Pathology Department in Mater Dei Hospital Malta were assessed. Furthermore, data was collected by reviewing the electronic medical records, histology results and imaging.

Result(s)* Over the 8 year period,146 new patients were diagnosed with advanced ovarian cancer. The median age at diagnosis was 64.5(33-91)years. 105/146(71.9\%) women presented with FIGO stage III and 41/146(28.1\%) with stage IV disease. Overall survival(OS) 32.87 months with a 1 -year survival of $70.55 \%$ and 5 -year survival of $20.28 \%$. Treatment was offered to $128 / 146(87.67 \%), \quad 103(80.47 \%)$ patients were offered 